\title{
Cytotoxic Constituents from the Leaves of Zanthoxylum schinifolium
}

\author{
Zhe Fang, Do Youn Jun, ${ }^{\dagger}$ Young Ho Kim, ${ }^{\dagger}$ Byung Sun Min, Ae Kyong Kim, and Mi Hee Woo* \\ College of Pharmacy, Catholic University of Daegu, Gyeongsan 712-702, Korea. *E-mail: woomh@cu.ac.kr \\ ${ }^{\dagger}$ Laboratory of Immunobiology, School of Life Science and Biotechnology, College of Natural Sciences, \\ Kyungpook National University, Daegu 702-701, Korea \\ Received March 6, 2009, Accepted February 17, 2010
}

Key Words: Zanthoxylum schinifolium, threo-6-Amino-5-hydroxy-5-methyl-1,3-oxazinan-4-one, Cytotoxicity, Cuspidiol, Auraptene

Several coumarins, methylschimilenol, schimitrienin, schininallylone, isoschinilenol, hydroxyepoxy-collinin, umbelliferone, ${ }^{1}$ alkaloid, benzenoid, chlorophylls, triterpenoid, diterpenoid, sesquiterpenoid, steroid, ${ }^{2}$ lignan, collinin, ${ }^{3}$ epoxyaurapten, hydrangetin, umbelliferone, acetoxycollinin, dictamine, norchelerythrine, skimmianine, $\beta$-amyrin, ${ }^{4}$ scoparone, schinifoline, scopoletin ${ }^{5}$ and xanthoxylin ${ }^{6}$ have been isolated from Zanthoxylum schinifolium (Rutaceae). Biological activities such as anti-diabetes, ${ }^{7}$ monoamine oxidase inhibition, ${ }^{8}$ anti-platelet, ${ }^{4}$ lipid peroxidation inhibition, ${ }^{9}$ anti-HBV DNA replication, ${ }^{3}$ antioxidant, ${ }^{5}$ insecticides, and nitrite-scavenging ${ }^{10}$ have been reported from this plant.

The roots, stems, pericarps, and seeds of $Z$. schinifolium were each extracted with $\mathrm{MeOH}$, and the leaves were extracted with $80 \% \mathrm{MeOH}$ and concentrated. These extracts were examined on MTT for cytotoxicity against Jurkat T cell clone E6.1. The results showed that the leaves extract had the strongest MTT cytotoxicity. The $\mathrm{MeOH}$ extract of $Z$. schinifolium leaves was subsequently fractionated into four parts: methylene chloride, ethyl acetate, $n$-butanol and water. These fractions were examined on MTT for cytotoxicity. The results showed that the methylene chloride fraction exhibited the strongest MTT cytotoxicity. Chromatographic separation of the methylene chloride and butanol fractions had yielded a quinolin (1), three phenylpropanoids $(\mathbf{2}, \mathbf{3}, \mathbf{1 2})$, four coumarins $(4 \sim 7)$, three triterpenoids $(8 \sim 10)$, an alkaloid (11), an alcohol glucoside (13) and three monoterpene glucosides $(\mathbf{1 4}, \mathbf{1 5}, \mathbf{1 6})$. One of these compounds were identified as new threo-6-amino-5-hydroxy-5-methyl1,3-oxazinan-4-one (11) together with fifteen known, 3-heptyl2-methylisoquinolin-1(2H)-one (1), integrifoliodiol (2), cuspidiol (3), bergapten (4), aurapten (5), 8-hydroxy-7-methoxychromen-2-one (6), 6,7-dimethoxy-2H-naphthalen-1-one (7), lupeol (8), lupeone (9), $\beta$-sitosterol (10), syringin (12), 2-propyl alchol $\beta$-D-glucopyranoside (13), vomifoliol-9- $O$ - $\beta$-D-glucopyranoside (14), betulalbuside A (15) and cnidioside C (16) on the basis of spectroscopic and chemical evidences. All of the compounds were isolated for the first time from this plant except 5 and 7. In the MTT cytotoxicity assay against Jurkat T cell clone E6.1, $\mathrm{IC}_{50}$ values of cuspidiol (3) and auraptene (5) were obtained at $7.3 \mu \mathrm{g} / \mathrm{mL}$ and $16.5 \mu \mathrm{g} / \mathrm{mL}$, respectively.

\section{Experimental Section}

Plant material. The leaves of Zanthoxylum schinifolium were collected in July 2004 from Gyeongsan, Gyeongbuk, Korea.

General experimental procedures. Mps were determined on a Yanaco micro melting point apparatus and were uncorrected. Optical rotations were taken on a Jasco DIP-370 digital polarimeter. IR spectra were measured on a Jasco FT/IR 300E spectrophotometer. UV spectra were obtained on a Shimadzu UV-160A spectrophotometer. ${ }^{1} \mathrm{H},{ }^{13} \mathrm{C}$ and $2 \mathrm{DNMR}$ spectra were performed on a Varian OXFORD AS400 MHz instrument. Spectrometry was measured in $\mathrm{CDCl}_{3}$ or $\mathrm{CD}_{3} \mathrm{OD}$ using TMS as an internal standard. Low- and high-resolution FABMS data were collected on a JMS-700 spectrometer. EIMS were recorded on a Quattro II spectrometer. For TLC, silica gel 60 F-254 (EM 5717) glass plates $(0.25 \mathrm{~mm})$ were used and visualized by spraying with $10 \%$ sulfuric acid in $\mathrm{H}_{2} \mathrm{O}$ and heating.

MTT cytotoxicity assay. The cytotoxic effect of compounds $\mathbf{1} \sim \mathbf{1 6}$ on Jurkat T cells clone E6.1 was analyzed by a 3-(4,5-dimethylthiazole-2-yl)-2,5-biphenyl tetrazolium bromide (MTT) assay reflecting cell viability, as described elsewhere. ${ }^{11}$ For the MTT assay, Jurkat T cells clone E6.1 $\left(5 \times 10^{4}\right.$ per well) were added to a serial dilution of compounds in 96 -well plates. After incubation for $44 \mathrm{~h}, 50 \mu \mathrm{L}$ of the MTT solution $(1.1 \mathrm{mg} / \mathrm{mL})$ was added to each well and incubated for an additional $4 \mathrm{~h}$. The colored formazan crystal produced from the MTT was dissolved in $150 \mu \mathrm{L}$ of dimethyl sulfoxide (DMSO). The optical density (OD) values of the solutions were measured at $540 \mathrm{~nm}$ using a plate reader.

Extraction and isolation. The leaves of Z. schinifolium (30 $\mathrm{kg}$ ) were freeze-dried and powdered to yield the leaves powder $10 \mathrm{~kg}$. The powder was extracted with $80 \% \mathrm{MeOH}$ to yield $1,206 \mathrm{~g}$ of an extract upon removal of the solvent. It was partitioned between $\mathrm{CH}_{2} \mathrm{Cl}_{2}-\mathrm{H}_{2} \mathrm{O}(1: 1)$ to yield the $\mathrm{CH}_{2} \mathrm{Cl}_{2}$-soluble fraction $(311 \mathrm{~g})$ and $\mathrm{H}_{2} \mathrm{O}$ solvent. The $\mathrm{H}_{2} \mathrm{O}$ solvent was partitioned with $n-\mathrm{BuOH}-\mathrm{H}_{2} \mathrm{O}(1: 1)$ to yield the $n$ - $\mathrm{BuOH}$-soluble fraction ( $385 \mathrm{~g}$ ) and the $\mathrm{H}_{2} \mathrm{O}$ soluble fraction (492 g). In the all fractions, $\mathrm{CH}_{2} \mathrm{Cl}_{2}$ fraction showed the strongest MTT cytotoxicity. The $\mathrm{CH}_{2} \mathrm{Cl}_{2}$-soluble fraction ( $150 \mathrm{~g}$ ) was subjected to open flash column chromatography over silica gel (200 g) eluted with hexane-EtOAc and $\mathrm{CH}_{2} \mathrm{Cl}_{2}-\mathrm{MeOH}$ gradient. Fractions (SL-MC-A to SL-MC-W) were collected and pooled according to their similar TLC patterns. Fraction SL-MC-R was chromatographed on a reverse-phase column $(60 \times 3.0 \mathrm{~cm}$, RP-C18) with $\mathrm{MeOH}-\mathrm{H}_{2} \mathrm{O}$ (gradient from 60:40 to 80:20) to afford compounds 1, 2 and 3. Fractions SL-MC-J, -D and -H were recrystallized to afford three compounds, 4,8 and 9 , respectively. Fraction 
Table 1. Cytotoxicity ( $\mathrm{IC}_{50}$ value) of $\mathbf{1} \sim \mathbf{1 6}$ on Jurkat $\mathrm{T}$ cells clone E6.1

\begin{tabular}{cc}
\hline Compound & $\mathrm{IC}_{50}(\mu \mathrm{g} / \mathrm{mL})$ \\
\hline $\mathbf{1}$ & $>50.0$ \\
$\mathbf{2}$ & $>50.0$ \\
$\mathbf{3}$ & 7.3 \\
$\mathbf{4}$ & $>50.0$ \\
$\mathbf{5}$ & 16.5 \\
$\mathbf{6}$ & $>50.0$ \\
$\mathbf{7}$ & $>50.0$ \\
$\mathbf{1 1}$ & $>50.0$ \\
$\mathbf{1 2}$ & $>50.0$ \\
$\mathbf{1 3}$ & $>50.0$ \\
$\mathbf{1 4}$ & $>50.0$ \\
$\mathbf{1 5}$ & $>50.0$ \\
$\mathbf{1 6}$ & $>50.0$ \\
\hline
\end{tabular}

SL-MC-I was recrystallized to afford two compounds, 5 and 10. Fraction SL-MC-O was chromatographed on a reversephase column $\left(60 \times 3.0 \mathrm{~cm}\right.$, Sephadex LH-20) with $\mathrm{MeOH}-\mathrm{H}_{2} \mathrm{O}$ (gradient from 65:35 to 95:5) to fractionate seven parts: SLMC-O-1 SL-MC-O-7. Fraction SL-MC-O-7 was chromatographed on a silica-phase column $(60 \times 3.0 \mathrm{~cm})$ with $\mathrm{CHCl}_{3}$ $\mathrm{MeOH}-\mathrm{H}_{2} \mathrm{O}$ (90:10:0.1) to afford compound 6. Fraction SLMC-O-4 was chromatographed on a reverse-phase column $(60 \times 3.0 \mathrm{~cm}, \mathrm{RP}-\mathrm{C} 18)$ with $\mathrm{MeOH}-\mathrm{H}_{2} \mathrm{O}(65: 35)$ to afford compound 7.

The butanol fraction (385 g) was subjected to open flash column chromatography over silica gel (400 g) eluted with hexane- $\mathrm{CH}_{2} \mathrm{Cl}_{2}$ and $\mathrm{CH}_{2} \mathrm{Cl}_{2}-\mathrm{MeOH}$ gradient. Fractions, (SL$\mathrm{Bu}-\mathrm{A}$ to SL-Bu-W) were collected and pooled according to their similar TLC patterns. Fraction SL-Bu-C was chromatographed on a reverse-phase column $(50 \times 2.5 \mathrm{~cm}$, RP-C18) with $\mathrm{MeOH}-\mathrm{H}_{2} \mathrm{O}$ (gradient from 18:82 to $90: 10$ ) to afford SLBu-C-A and SL-Bu-C-B. SL-Bu-C-A was chromatographed<smiles>CCCCCCCc1cc(=O)c2ccccc2n1C</smiles>
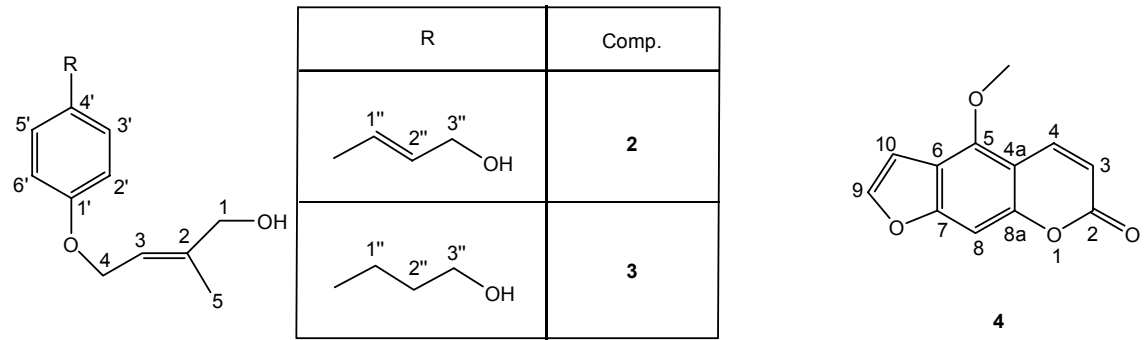<smiles>[R]c1cc2ccc(=O)oc2c([R])c1[2H]</smiles>

\begin{tabular}{|c|c|c|c|}
\hline $\mathrm{R}_{1}$ & $\mathrm{R}_{3}$ & Comp. \\
\hline$-\mathrm{H}$ & $\mathrm{R}_{6^{\prime}}^{\mathrm{R}_{2}}$ & $-\mathrm{H}$ & $\mathbf{5}$ \\
\hline$-\mathrm{H}$ & $\mathrm{C}^{\prime} \mathrm{OCH}_{3}$ & $-\mathrm{OH}$ & $\mathbf{6}$ \\
\hline $\mathrm{OCH}_{3}$ & $-\mathrm{OCH}_{3}$ & $-\mathrm{H}$ & $\mathbf{7}$ \\
\hline
\end{tabular}
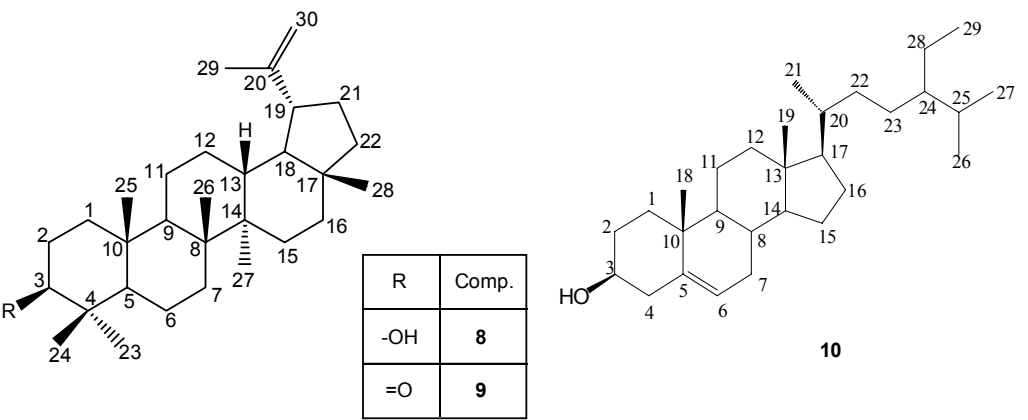

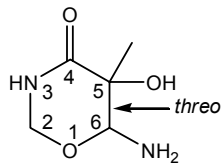

11

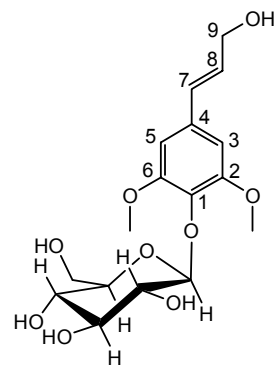

12

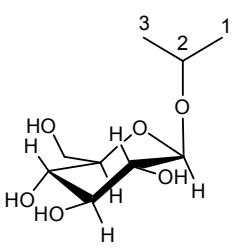

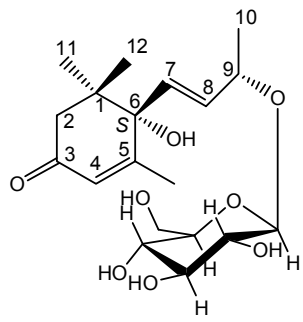

14

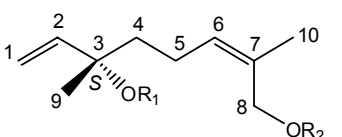

\begin{tabular}{|c|c|c|}
\hline$R_{1}$ & $R_{2}$ & Comp. \\
\hline$-H$ & -gluc. & 15 \\
\hline -gluc. & $-H$ & 16 \\
\hline
\end{tabular}

Figure 1. Structures of compounds isolated from methylene chloride $(\mathbf{1} \sim \mathbf{1 0})$ and butanol $(\mathbf{1 1} \sim \mathbf{1 6})$ fractions of Z. schinifolium. 
on a silica-phase column $(45 \times 2.0 \mathrm{~cm})$ with $\mathrm{CHCl}_{3}-\mathrm{MeOH}$ (15:1) to afford compound 11. SL-Bu-C-B was chromatographed on a silica-phase column $(40 \times 1.8 \mathrm{~cm})$ with $\mathrm{CHCl}_{3}-\mathrm{MeOH}$ (20:1) to afford compound 12. Fraction SL-Bu-F was chromatographed on a reverse-phase column $(60 \times 3.0 \mathrm{~cm}$, RPC18) with $\mathrm{MeOH}-\mathrm{H}_{2} \mathrm{O}$ (gradient from 1:100 to 85:15) to fractionate six parts: SL-Bu-F-A $\sim$ SL-Bu-F-F. SL-Bu-F-A was chromatographed on a silica-phase column $(40 \times 2.0 \mathrm{~cm})$ with $\mathrm{CHCl}_{3}-\mathrm{MeOH}$ (18:1) to afford compound 13. SL-Bu-F-C was chromatographed on a silica-phase column $(38 \times 1.5 \mathrm{~cm})$ with $\mathrm{CHCl}_{3}-\mathrm{MeOH}$ (20:1) to afford compound 14. SL-Bu-F-E was chromatographed on a silica-phase column $(40 \times 1.8 \mathrm{~cm})$ with $\mathrm{CHCl}_{3}-\mathrm{MeOH}(25: 1)$ to afford compound 15. SL-Bu-F-F was chromatographed on a silica-phase column $(40 \times 2.0 \mathrm{~cm})$ with $\mathrm{CHCl}_{3}-\mathrm{MeOH}(15: 1)$ to afford compound 16.

Integnifoliodiol (2): $[\alpha]_{\mathrm{D}}^{25}-7.0\left(c 0.2, \mathrm{CH}_{2} \mathrm{Cl}_{2}\right)$; UV (MC) $\lambda_{\max }$ $(\log \varepsilon) 266.5$ (4.04) nm; IR (KBr) $v_{\max } 3293,2921,2854,1606$, $1457,1396,1022,802 \mathrm{~cm}^{-1}$; EIMS $m / z 234$ [M] ${ }^{+}$; FABMS $m / z$ : $235.1[\mathrm{M}+\mathrm{H}]^{+}$; HRFABMS $m / z 235.1333$ (calc. 233.2334 for $\left.\mathrm{C}_{14} \mathrm{H}_{18} \mathrm{O}_{3}\right) ;{ }^{1} \mathrm{H}-\mathrm{NMR}\left(400 \mathrm{MHz}, \mathrm{CDCl}_{3}, \delta_{\mathrm{H}}\right) 7.32(2 \mathrm{H}, \mathrm{d}, J=$ $\left.8.4 \mathrm{~Hz}, \mathrm{H}-3^{\prime}, 5^{\prime}\right), 6.87\left(2 \mathrm{H}, \mathrm{d}, J=8.4 \mathrm{~Hz}, \mathrm{H}-2^{\prime}, 6^{\prime}\right), 6.55(1 \mathrm{H}, \mathrm{d}$, $\left.J=16.0 \mathrm{~Hz}, \mathrm{H}-1^{\prime \prime}\right), 6.24\left(1 \mathrm{H}, \mathrm{dt}, J=16.0,6.0 \mathrm{~Hz}, \mathrm{H}-2^{\prime \prime}\right), 5.77$ $(1 \mathrm{H}, \mathrm{dt}, J=7.2,1.6 \mathrm{~Hz}, \mathrm{H}-3), 4.60(2 \mathrm{H}, \mathrm{d}, J=7.2 \mathrm{~Hz}, \mathrm{H}-4), 4.30$ $\left(2 \mathrm{H}, \mathrm{dd}, J=6.0,1.2 \mathrm{~Hz}, \mathrm{H}-3^{\prime \prime}\right), 4.09$ (2H, s, H-1), 1.77 (3H, s, $\mathrm{H}-5) ;{ }^{13} \mathrm{C}-\mathrm{NMR}\left(100 \mathrm{MHz}, \mathrm{CDCl}_{3}, \delta_{\mathrm{C}}\right) 158.6\left(\mathrm{C}-1^{\prime}\right), 140.3$ (C-2), $131.2\left(\mathrm{C}-1^{\prime \prime}\right), 129.7\left(\mathrm{C}-4^{\prime}\right), 127.9$ (C-3', 5'), 126.5 (C$\left.2^{\prime \prime}\right), 120.1$ (C-3), 114.7 (C-2', 6'), 68.0 (C-1), 64.6 (C-4), 64.1 (C-3"), 14.3 (C-5).

threo-6-Amino-5-hydroxy-5-methyl-1,3-oxazinan-4-one (11): $[\alpha]_{\mathrm{D}}^{25} 20.1(c 0.10, \mathrm{MeOH}) ; \mathrm{UV}(\mathrm{MeOH}) \lambda_{\max }(\log \varepsilon)$ 206.5 (3.86) nm; IR (KBr) 3427, 2928, 2982, 1775, 1638, 1474, 1210, 1108, $1033 \mathrm{~cm}^{-1}$; FABMS $m / z$ 147.1 [M+H] $]^{+}, 73.2,55.3$; HRFABMS $m / z 147.0770$ (calc. 147.0765 for $\mathrm{C}_{5} \mathrm{H}_{11} \mathrm{O}_{3} \mathrm{~N}_{2}$ ); ${ }^{1} \mathrm{H}-\mathrm{NMR}\left(400 \mathrm{MHz}, \mathrm{CD}_{3} \mathrm{OD}, \delta_{\mathrm{H}}\right) 4.37(1 \mathrm{H}, \mathrm{dd}, J=10.8,3.6$ $\mathrm{Hz}, \mathrm{H}-2 \mathrm{a}), 4.33$ (1H, dd, $J=10.8,1.2 \mathrm{~Hz}, \mathrm{H}-2 \mathrm{~b}), 4.15$ (1H, dd, $J=3.6,1.2 \mathrm{~Hz}, \mathrm{H}-6), 1.48\left(3 \mathrm{H}, \mathrm{s}, \mathrm{H}-\mathrm{CH}_{3}\right) ;{ }^{13} \mathrm{C}-\mathrm{NMR}(100 \mathrm{MHz}$, $\left.\mathrm{CD}_{3} \mathrm{OD}, \delta_{\mathrm{C}}\right) 178.3(\mathrm{C}-4), 73.8(\mathrm{C}-5), 73.4$ (C-6), 72.3 (C-2), $21.8\left(\mathrm{C}-\mathrm{CH}_{3}\right)$.

\section{Results and Discussion}

The leaves of $Z$. schinifolium were extracted with $80 \% \mathrm{MeOH}$. The $\mathrm{MeOH}$ extract was subsequently fractionated into four parts: $\mathrm{CH}_{2} \mathrm{Cl}_{2}$, EtOAc, $n$-BuOH and $\mathrm{H}_{2} \mathrm{O}$. From the MTT cytotoxicity-directed fractionation, we have isolated ten known compounds $(\mathbf{1} \sim \mathbf{1 0})$ from the $\mathrm{CH}_{2} \mathrm{Cl}_{2}$ fraction and a new (11) and five known compounds $(\mathbf{1 2} \sim \mathbf{1 6})$ from butanol fraction by $\mathrm{SiO}_{2}$ flash, RP-C18 and $\mathrm{SiO}_{2}$ open, and Sephadex LH-20 column chromatographies.

Compound 11 was found to be new. Known compounds $1 \sim$ 10 and $12 \sim 16$ were determined by direct comparison with authentic samples or by comparing their physical and spectral data with those in the literature: 3-heptyl-2-methylisoquinolin1(2H)-one ${ }^{12}$ (1), 4-[4-(3-hydroxy-propenyl)-phenoxy]-2-methylbut-2-en-1-ol (integrifoliodiol, ${ }^{13}$ 2), 4-[4-(3-hydroxy-propyl)phenoxy]-2-methylbut-2-en-1-ol (cuspidiol, ${ }^{14} \mathbf{3}$ ), bergapten $^{15}$ (4), 7-[(3,7-dimethyl-2,6-octa-dienyl)oxy]-coumarin (auraptene, ${ }^{4}$ 5), 8-hydroxy-7-methoxy-chromen-2-one ${ }^{16}(6)$, 6,7-dimethoxy-
$2 H$-naphthalen-1-one ${ }^{17}$ (7), 20(29)-lupen-3-ol (lupeol, ${ }^{18} 8$ ), 20(29)-lupen-3-one (lupeone, ${ }^{19}$ 9), $\beta$-sitosterol ${ }^{20}(\mathbf{1 0})$, syringin ${ }^{21}$ (12), 2-propyl alchol $\beta$-D-glucopyranoside (13), vomifoliol$9-O-\beta$-D-glucopyranoside ${ }^{22}(14)$, betulalbuside $\mathrm{A}^{23}(\mathbf{1 5})$ and cnidioside $\mathrm{C}^{24}(\mathbf{1 6})$. All of the compounds were isolated for the first time from this plant except 5, 7 and $\mathbf{1 0}$.

FABMS spectral analysis of compound $\mathbf{1 1}$ showed a $[\mathrm{M}+\mathrm{H}]^{+}$ at $\mathrm{m} / z$ 147.1. HRFABMS spectral analysis of $\mathbf{1 1}$ showed a $[\mathrm{M}+\mathrm{H}]^{+}$at $m / z 147.0770$ (calc. 147.0765) which corresponded to the molecular formula $\mathrm{C}_{5} \mathrm{H}_{11} \mathrm{O}_{3} \mathrm{~N}_{2}$. The IR spectrum showed absorption bands at $3426 \mathrm{~cm}^{-1}(\mathrm{OH}), 1775(\mathrm{C}=\mathrm{O})$, and 1108 $\mathrm{cm}^{-1}(\mathrm{C}-\mathrm{O})$. The ${ }^{1} \mathrm{H}-\mathrm{NMR}$ spectrum of $\mathbf{1 1}$ afforded signals for a methyl proton as singlet at $\delta 1.48(3 \mathrm{H})$. Two doublet of doublet proton peaks were present at $\delta 4.37(1 \mathrm{H}, \mathrm{dd}, J=10.8$, $3.6 \mathrm{~Hz})$ and $4.33(1 \mathrm{H}, \mathrm{dd}, J=10.8,1.2 \mathrm{~Hz})$ integrating for two protons $\mathrm{H}-2 \mathrm{a}$ and $\mathrm{H}-2 \mathrm{~b}$. That spectrum also indicated a signal at $\delta 4.15(1 \mathrm{H}, \mathrm{dd}, J=3.6,1.2 \mathrm{~Hz})$ which could be assigned to $\mathrm{H}-6$. The ${ }^{13} \mathrm{C}-\mathrm{NMR}$ spectrum of $\mathbf{1 1}$ indicated the presence of a ketone carbon and a methyl carbon signals at $\delta 178.3$ (C-4) and $21.8\left(\mathrm{C}-\mathrm{CH}_{3}\right)$, respectively. A hydroxylated carbon was exhibited at $\delta 73.8(\mathrm{C}-5)$. Two oxygen connected carbons, $\mathrm{C}-2$ and $\mathrm{C}-6$, were exhibited at $\delta 72.3$ and 73.4 , respectively. The proton and carbon signals were assigned with the help of HSQC, $\mathrm{HMBC}$ and COSY experiments. In the HMBC spectrum of 11, the correlation displayed connectivities between $\mathrm{C}-4$ and $\mathrm{H}-2$, $\mathrm{H}-6$ and $\mathrm{H}-\mathrm{CH}_{3}$, between $\mathrm{C}-6$ and $\mathrm{H}-2 \mathrm{ab}$ and $\mathrm{H}-\mathrm{CH}_{3}$, between $\mathrm{C}-2$ and $\mathrm{H}-6$, and between $\mathrm{C}-5$ and $\mathrm{H}-6$ and $\mathrm{H}-\mathrm{CH}_{3}$. The correlation in the COSY spectrum displayed connectivities between $\mathrm{H}-2$ and $\mathrm{H}-6$, between $\mathrm{H}-6$ and $\mathrm{H}-\mathrm{CH}_{3}$. Irradiation of the proton $\mathrm{H}-\mathrm{CH}_{3}$ at $\delta 1.48$ showed a positive NOE effect with $\delta 4.15$ (H-6), thus the relative stereochemistry between C-5 and C-6 was determined as threo. Therefore, the structure of compound 11 was exhibited as threo-6-amino-5-hydroxy-5-methyl-1,3oxazinan-4-one.

In the MTT cytotoxicity assay against Jurkat T cell clone E6.1, IC I0 $_{50}$ values of cuspidiol (3) and auraptene (5) were obtained at $7.3 \mu \mathrm{g} / \mathrm{mL}$ and $16.5 \mu \mathrm{g} / \mathrm{mL}$, respectively. Cytotoxicity for $\mathbf{8} \sim \mathbf{1 0}$ could not be available because of their insolubility in DMSO.

Acknowledgments. The authors are grateful to S. H. Kim and collaborators at the Korea Basic Science Institute (Daegu) for measuring the mass spectra. This work was supported by the Technology Development Program for Agriculture and Forestry, Ministry of Agriculture and Forestry, Republic of Korea (Grant No. 203008-03-2-HD110).

\section{References}

1. Tsai, L. L.; Lin, W. Y.; Teng, C. M. Planta Med. 2000, 66, 618.

2. Cheng, C. M.; Yang, C. H.; Lin, W. Y. J. Chin. Chem. Soc. 2001, 49,125 .

3. Chang, C. T.; Doong, S. L.; Tasi, I. L. Phytochemistry 1997, 45, 1419.

4. Chen, I. S.; Lin, Y. C.; Tsai, I. L. Phytochemistry 1995, 39, 1091.

5. Pan, J. X.; Bai, Q.; Yang, B. Zhiwu Ziyuan Yu Huanjing 1993, 2, 26.

6. Liu, S. L.; Wei, L. X. Zhongcaoyao 1991, 22, 16.

7. Kim, S. H.; Kwon, C. S.; Lee, J. S. J. Food Sci. Nutr. 2002, 7, 62.

8. Jo, Y. S.; Hong, D. T. L.; Bae, K. H. Planta Med. 2002, 68, 84.

9. Mun, S. I.; Ryu, H. S.; Choi, S. J. Korean J. Food \& Nutr. 1997, 
26,943.

10. Kim, D. S.; Ahn, B. W.; Yeum, D. M. J. Korean Fish. Soc. 1987, 20, 463.

11. Jun, D. Y.; Rue, S. W.; Han, K. H.; Taub, D.; Lee, Y. S.; Bae, Y. S.; Kim, Y. H. Biochem. Pharmacol. 2003, 66, 2291.

12. Yuan, Q. T.; Xiao, Z. F.; Liang, H. Phytochemistry 1996, 43, 719.

13. Cheng, M. J.; Lin, C. F.; Wang, C. J.; Tsai, I. L.; Chen, I. S. J. Chin. Chem. Soc. 2007, 54, 779.

14. Ishii, H.; Ishikawa, T.; Teh, L. S. Yakugaku Zasshi 1976, 96, 1458.

15. Sasaki, H.; Taguchi, H. C.; Endo, T. Chem. Pharm. Bull. 1982, 30, 3555.

16. Barua, N. C.; Sharma, R. P.; Madhusudanan, K. P. Phytochemistry 1980, 19, 2217.
17. Ulubelen, A.; Oksuz, S. J. Nat. Prod. 1984, 47, 170.

18. Sholichin, M.; Yamasaki, K.; Kasai, R. Chem. Pharm. Bull. 1980, 28,1006 .

19. Ohmoto, T. Yakugaku Zasshi 1969, 89, 814.

20. Gella, M. D.; Monako, P.; Previtera, L. J. Nat. Prod. 1990, 53, 1430.

21. Joanna, C.; Gerard, N.; Wanda, K. Biochem. Syst. Ecol. 2006, 34 , 862.

22. Anni, P.; Denis, B.; Etienne, S.; Peter, S. Phytochemistry 1992, 31,1649

23. Lalcin, F. N.; Ersoz, T.; Akbay, P.; Calis, I. Turk. J. Chem. 2003, 27,703 .

24. Taketo, U.; Toshio, M.; Akira, U.; Khan, U. Phytochemistry 1989, 28, 3369 . 